\title{
Find It - An Assistant Home Agent
}

Angelo Costa, Ester Martinez-Martin, Angel P. del Pobil, Ricardo Simoes and Paulo Novais

\begin{abstract}
Cognitive impaired population face with innumerable problems in their daily life. Surprisingly, they are not provided with any help to perform those tasks for which they have difficulties. As a consequence, it is necessary to develop systems that allow those people to live independently and autonomously. Living in a technological era, people could take advantage of the available technology, being provided with some solutions to their needs. This paper presents a platform that assists users with remembering where their possessions are. Mainly, an object recognition process together with an intelligent scheduling applications are integrated in an Ambient Assisted Living (AAL) environment.
\end{abstract}

\section{Introduction}

Countries and societies evolve at a high rate leading to new social problems to be dealt with. One of these problems is related to the population undergrowth in developed countries. The reason for that undergrowth lies on the fact that birth rate is continuously decreasing, while life expectancy is increasing. For that reason, from

Angelo Costa and Paulo Novais

CCTC - Computer Science and Technology Center, Department of Informatics, University of Minho, Braga, Portugal e-mail: acosta@di.uminho.pt, pjon@di.uminho.pt

Ester Martinez-Martin and Angel P. del Pobil

Robotic Intelligence Lab, Engineering and Computer Science Department, Jaume-I University, Castellón, Spain e-mail: emartine@uji.es, pobil@uji.es

Ricardo Simoes

Institute of Polymers and Composites IPC/I3N, University of Minho, Guimarães, Portugal; Life and Health Sciences Research Institute (ICVS), School of Health Sciences, University of Minho, Campus de Gualtar, 4710-057 Braga, Portugal; Polytechnic Institute of Cávado and Ave, Barcelos, Portugal e-mail: rsimoes@dep.uminho.pt 
the current society model, two problems arise: medical response and economic stability.

Speaking in terms of cognitive problems, the increase in elderly people implies that the common problems affecting them, grow in incidence. Regarding economy, country production has a severe setback: population cannot get the necessary income to support everyone. Therefore, expensive services are beyond most of the population.

In addition, the cognitive problems are very crippling in terms of independence. What is more, these problems can lead to other health problems. So, for instance, it is common to lose balance and, consequently, fall down. This fact could result in broken bones and other diseases that can affect severely the elderly life, resulting even in death (in extreme cases). In terms of daily life, a person needs constant attention when the cognitive impairment is severe. That attention can be provided by a personal caregiver or by living at a specialized institution. Consequently, these people need little help in their everyday activities, allowing them to live alone, as well as more cognitive aids like refreshing their memories, are required.

Elderly population does still not have all the help they need. For that, designing an agent aiding people to remember where their possessions are at home is imperative. This paper addresses the general directions for the design and implementation of an intelligent agent aimed at this issue. We present a modular architecture such that, from visual information gathered at home by a set of cameras, the agent will provide the user with the location of their most common possessions like their keys.

\section{General directions of AAL-Related Research}

Ambient Intelligence can be defined as the research focused on sensitive and responsive electronic environments, involving the user. That is, it is aimed to provide people with technological solutions to help them from perceiving and analyzing their environment. As part of the AmI area, we can find the Ambient Assisting Living (AAL). In particular, the AmI covers all the electronic devices, while the AAL is focused on any user with disabilities and his/her home.

Despite the AAL progress, it is still a diffuse area because each project is aimed at solving a particular task, but not at being part of a whole integrated environment of services. Consequently, the lack of cooperation between the developed projects results in a group of services that do not communicate each other, loosing precious features that could outcome from projects' fusion. Furthermore, without well-established standards, it is nearly impossible to integrate different projects into a more complete, practical system. On this matter, Norgall et al. [1] analyzed the latest developments and standards on this topic. In this study, they proposed some advices on development as well as future global standards. Among them, it should be pointed out the architecture, communication and physical implementation. The AAL4ALL project is a pioneer on this topic. It is a consortium of technological and medical partners that work on producing and implementing complete AAL 
platforms. These platforms can be used in user's home, in a hospital or at a daycentre [2]. The multi-agent system is able to connect different developments in the same platform, integrating them in a unified service. So, information can be exchanged and linked resulting in more complex and sophisticated services [3].

From this starting point and keeping in mind the user's needs, we propose a complete, modular system to assist a user in remembering his/her activities as well as all the required possessions before leaving his/her home (e.g. home keys) and where they are, if necessary. With that aim, we present a multi-agent home platform where a vision module for object recognition and location is integrated. So, the implemented multi-agent platform is presented in Section 3, while the vision module is described in Section 4. Finally, some conclusions and future work is discussed in Section 5 .

\section{Multi-Agent Home Platform}

The multi-agent home platform, which consists of a HAN (Home Area Network) and BAN (Body Area Network), monitors and helps a person to perform their daily tasks. Every module contains, at least, two agents: one for action control; and another for communication. Note that, in a further stage, some sensors could be attached to user clothes for capturing their body signs.

The iGenda project is one part of this system $[4,5]$. It offers a mobile system (on a Smartphone), in terms of interface, to the user, that is, it provides an intelligent schedule system and an appealing interface. The scheduling system handles new events sent from different users or platforms, and leisure events. In addition, a profiling system has been integrated to improve the decision module. The module is connected to the server framework for sending and receiving information about the operations and actions occurring within the platform, as shown in Fig. 1.

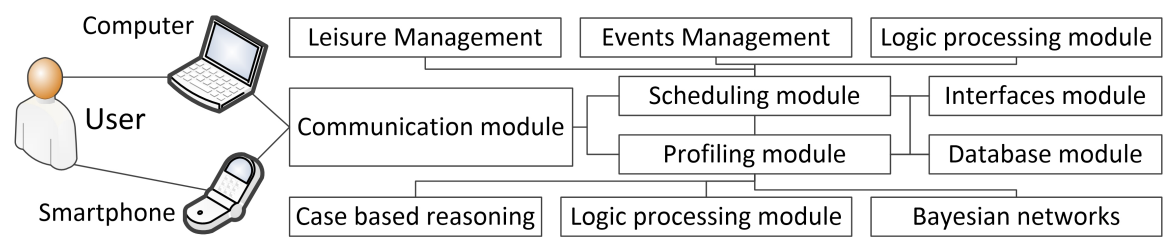

Fig. 1 iGenda modules and systems overview

Since the mobile platform is implemented over the Android operating system [6], it has full support to the Google services, including calendar, email and chat. With the aim of a successful performance, the system must be permanently active, even when there is no connection with the server. Therefore, the system is composed of different agents managing each feature. Mainly, these agents are divided into communications, logic decisions, interface bridging and database management. Among 
all these features, the profiling system is the most important to this project merging action.

The profiling system consists of an agent integrated in the scheduling module. This agent is in charge of controlling the module learning process by keeping all the information relative to user interactions. That is, all the received user edits and sends of events are registered in an event history [7]. Two types of learning systems are used to emulate the user preferences: Case Based Reasoning (CBR) and Bayesian Networks (BN) [8, 9].

With the aim of making the concept clearer, think about the traveling salesman problem. It has the same implementation foundations, evolving to a multi-level weighted graph. Thereon, heuristic compounds allow to creating probabilistic computational tree for each event. In terms of tree limitation or pruning, a trust value of $80 \%$ is used to constrain the fractal evolution. That is, the sum of three or less tree vectors must be above $80 \%$, where the vectors refer to the number of times an event has been used to modulate the user's decision. Another highlighting feature of the profiling agent is keeping the overall user's preferences since this information could be useful for other modules. For instance, tracking the common user's objects and their location makes easier to provide information about them to the user. Therefore, when an object is required for performing an activity, the object recognition system could be used to remind where it is.

The goal is to open a path to connect other projects with the iGenda. That is, opening a way to share the information that both projects have. As previously stated, our perspective relays on joining developments, not on building a new project. Due to the nature of the iGenda project and its aim, an object recognition feature is the best solution to aid the user locating objects. Thus, it can result in the following scenario: if the user needs to exit its home he/she has to take the house keys, thus, the platform can warn the user that the keys are needed and, at the same time, show where they are.

\section{Object recognition and location}

Object recognition is one of the most important problems in Computer Vision. For decades, researchers have introduced many algorithms in the hope of building systems with the ability of visually perceiving their surrounding environment. In recent years, with the advances in neuroscience and the discoveries about how the human vision system processes visual information, computer scientists got focused on mapping those findings to build computational models emulating human object recognition [10]. Some developed models as those based on the visual primate system [11], have produced admirable results. However, it could not perform well in some situations as cluttered scenarios and/or with partially occluded objects.

So, the issues to be solved can be summarized as: object recognition at any time; and object location within the whole home. Therefore, any time a user enters his/her home, the agent is surveilling his/her possessions such that they are recognized and 
tracked along the time. In that way, in the case the user forgets where he/she has left a certain possession, the developed module can accurately provide its location.

In this context, the first issue to be solved refers to object recognition. Basically, it implies two different tasks: object detection and object identification. So, on the one hand, object detection refers to the ability of identifying the presence/absence of certain objects in an image. Given its importance for a wide range of visual applications, research on this topic has taken a number of forms. For instance, several approaches have built models of the objects to be detected (e.g. [12, 13]). Mainly, these techniques consist of learning a model of the interest objects in advance from a large set of training images. These saved images show the object in different poses and from different viewpoints. Although it could be a good solution for some kind of objects, it is difficult to learn models of objects with a high dimensionality and/or with a rich variability in their motion. On the contrary, other approaches have been proposed depending on the considered visual features such as shape [14], colour [15, 16], texture [17], appearance [18], motion [19], patch [20] or gradient [21]. Nevertheless, it is difficult to design a robust method based on visual features when working on real scenarios where different factors such as occlusions, changes in illumination, different viewpoint, or dynamic backgrounds (e.g. waving trees, moving curtains, or blinking screens), can make them miserably fail. As a solution, background removal algorithms have been developed (e.g. [22, 23, 24]). Basically, a statistical background model, built after observing a scene during several seconds, is used to identify objects of interest. Nevertheless, these techniques present some drawbacks to be overcome such as everything seen when the background model is being built is considered background; no sudden changes in illumination occur during the whole experiment; an object which appeared in the scene and then stopped moving for a period of time can be modelled as a part of the background; and, the resulting binary image segmentation of the image only highlights regions of non-stationary objects.

As a consequence, a mechanism for object detection in real-life scenarios has to be deployed. Keeping in mind that objects to be identified are left to any place by a person, motion is considered the primary cue for object detection. That is, this method is based on the idea that a person is leaving their possessions while they are moving around their home. Therefore, we have implemented a new approach $[25,26]$ able of managing a training period with foreground objects, adapting to minor dynamic, uncontrolled changes (e.g. the passage of time, blinking of screens or shadows), adapting to sudden changes in illumination and distinguishing between target objects and background in terms of motion and motionless situations.

On the other hand, once objects are detected in a captured image, object identification is the next step. On this matter, the key issue is determining which characteristic visual information allows us to uniquely identify such target objects. For that reason, although motion allows the system to robustly detect possessions in an image sequence, other features must be used. Despite the wide variety of existing approaches based on visual features [27], they are inappropriate for our purpose because they provide a variable number of features to describe an object. Thus, a high number of features results in a high space-consuming application, whilst a low one 

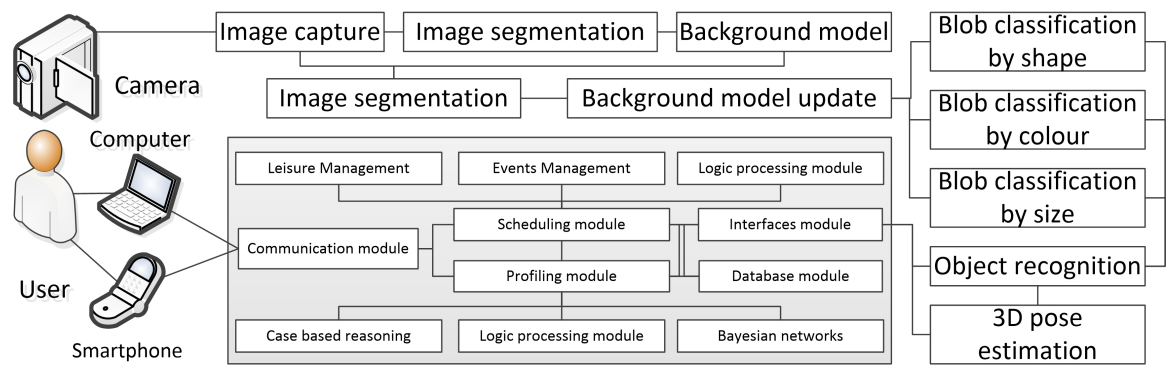

Fig. 2 iGenda and Object Recognition system overview

would provide inaccuracy. Moreover, the detection of substantial occlusion levels requires a large number of key points leading to a high computational cost. What is more, the relationship existing between the number of visual features and the number of objects to be tracked constrains the application. For that reason, in this work, shape, colour and size are used. Consequently, our object representation is composed of these three visual features that are modelled taking into account the variability of their values due to viewpoint changes and different illumination conditions.

The final stage of this process refers to object location within user's home. In this case, the geometry of multiple cameras is exploited to obtain the 3D object pose. Therefore, considering the most common lost objects (i.e. the keys, the wallet, the glasses, etc.), a distinctive representation of each of them is being designed and used by the visual agent. So, it can extract information about possession location from the visual input provided by a heterogeneous set of cameras composed of colour traditional and fisheye devices.

\section{Conclusions and Future Work}

In this paper, a modular system to aid people in remembering their scheduled tasks as well as all the objects required to perform those tasks, is presented. For that, a vision module for object recognition and location is integrated in a multi-agent home platform. This integration is based on the agent concept, which allows to overcoming the great difference between the module internal data-flow. In that way, the proposed system lays the foundations for a compliant AAL ecosystem aimed at assuring the fusion of singularly developed projects with the purpose of getting new features in different environments and platforms.

Therefore, on the one hand, the multi-agent home platform will handle the information about the requested object, searching relations in the user information database, and managing the user interfaces to accommodate new information. An aftereffect can be the introduction of a user's home map in the interface where the object in need can be easily located by showing in which part of the house it is. 
On the other hand, the vision module will recognize and locate the target objects. So, while object recognition considers object shape, colour and size from a segmented image based on a background model, the object location exploites the geometry of the camera set to properly estimate 3D object poses.

The future work will be in terms of developing the communication structures and the intergration with the scheduling system. New agents and modules must be built to process the new incoming data and the bilateral trade of information. The key issue of the development is that the current core systems of both modules do not have to be changed to receive the new agents. The technological aim is to change the least possible the developed systems and to implement new features. Therefore, validating the proposal of building a stable AAL platform establishes two different projects.

\section{Acknowledgments}

This work is partially funded by National Funds through the FCT-Fundação para a Ciencia e a Tecnologia (Portuguese Foundation for Science and Technology) within projects PEst-OE/EEI/UI0752/2011, and PEst-C/CTM/LA0025/2011.

Project AAL4ALL, co-financed by the European Community Fund FEDER through COMPETE - Programa Operacional Factores de Competitividade (POFC). Authors also want to thank the support received from the Ministerio de Ciencia e Innovación (DPI2011-27846), by Generalitat Valenciana (PROMETEO/2009/052) and by Fundació Caixa Castelló-Bancaixa (P1-1B2011-54).

\section{References}

1. Tazari, M.R., Wichert, R., Norgall, T.: Towards a unified ambient assisted living and personal health environment. In Wichert, R., Eberhardt, B., eds.: Ambient Assisted Living. Springer Berlin Heidelberg (2011) 141-155

2. Vardasca, R., Simoes, R.: Needs and opportunities in ambient assisted living in portugal. In: 2nd Int. Living Usability Lab Workshop on AAL Latest Solutions, Trends and Applications, AAL 2012, in Conjunction with BIOSTEC 2012. (2012) 100 - 108

3. Acampora, G., Loia, V.: A dynamical cognitive multi-agent system for enhancing ambient intelligence scenarios. In: IEEE Int. Conf. on Fuzzy Systems. (2009) 770-777

4. Costa, A., Castillo, J.C., Novais, P., Fernández-Caballero, A., Simoes, R.: Sensor-driven agenda for intelligent home care of the elderly. Expert Systems with Applications 39(15) (2012) 12192-12204

5. Costa, A., Novais, P., Corchado, J.M., Neves, J.: Increased performance and better patient attendance in an hospital with the use of smart agendas. Logic Journal of IGPL 20(4) (2012) 689-698

6. Ughetti, M., Trucco, T., Gotta, D.: Development of agent-based, peer-to-peer mobile applications on android with jade. In: The 2nd Int. Conf. on Mobile Ubiquitous Computing, Systems, Services and Technologies. (2008) 287-294

7. Marques, V., Costa, A., Novais, P.: A dynamic user profiling technique in a AmI environment. In: World Congress on Information and Communication Technologies, IEEE (2011) 1247 1252 
8. Radde, S., Freitag, B.: Using Bayesian Networks To Infer Product Rankings From User Needs. In: UMAP 2010 Workshop on Intelligent Techniques for Web Personalization and Recommender Systems. (2010)

9. Watson, I.: An introduction to case-based reasoning. In Watson, I., ed.: Progress in CaseBased Reasoning. Volume 1020 of LNCS. Springer Berlin / Heidelberg (1995) 1-16

10. Guido, D.: AP Neuroscience Course (2011)

11. Serre, T., Wolf, L., Poggio, T.: Object recognition with features inspired by visual cortex. In: CVPR. Volume 2. (2005) 994-1000

12. Cristinacce, D., Cootes, T.F.: Boosted regression active shape models. In: British Machine Vision Conference. Volume 2. (2007) 880-889

13. Urtasun, R., Fleet, D.J., Fua, P.: Temporal motion models for monocular and multiview 3d human body tracking. CVIU 104(2-3) (2006) 157-177

14. Toshev, A., Taskar, B., Daniilidis, K.: Shape-based object detection via boundary structure segmentation. IJCV 99(2) (2012) 123-146

15. Shahbaz Khan, F., Anwer, R.M., van de Weijer, J., Bagdanov, A.D., Vanrell, M., Lopez, A.M.: Color attributes for object detection. In: CVPR. (2012) 3306-3313

16. Urdiales, C., Dominguez, M., de Trazegnies, C., Sandoval, F.: A new pyramid-based color image representation for visual localization. Image and Vision Computing 28(1) (2010) 7891

17. Bileschi, S.M., Wolf, L.: A unified system for object detection, texture recognition, and context analysis based on the standard model feature set. In: British Machine Vision Conference. Volume 83. (2005) 1-10

18. Borotschnig, H., Paletta, L., Prantl, M., Pinz, A.: Appearance-based active object recognition. Image and Vision Computing 18(9) (2000) 715-727

19. Ciliberto, C., Pattacini, U., Natale, L., Nori, F., Metta, G.: Reexamining lucas-kanade method for real-time independent motion detection: Application to the icub humanoid robot. In: IEEE/RSJ Int. Conf. on Intelligent Robots and Systems. (2011) 4154-4160

20. Keysers, D., Deselaers, T., Breuel, T.M.: Optimal geometric matching for patch-based object detection. ELCVIA 6(1) (2007) 44-54

21. Dalal, N., Triggs, B.: Histograms of oriented gradients for human detection. In: CVPR. Volume 1. (2005) 886-893

22. Ahmed, S., El-Sayed, K., Elhabian, S.: Moving object detection in spatial domain using background removal techniques - state-of-art. Recent Patents on Computer Sciencee 1(1) (2008) 32-54

23. Varcheie, P.D.Z., Sills-Lavoie, M., Bilodeau, G.A.: An efficient region-based background subtraction technique. In: Canadian Conference on Computer and Robot Vision. (2008) 7178

24. Migliore, D.A., Matteucci, M., Naccari, M.: A revaluation of frame difference in fast and robust motion detection. In: 4th ACM Int. Workshop on Video surveillance and sensor networks - VSSN '06, New York, New York, USA, ACM Press (2006) 215

25. Martinez-Martin, E., del Pobil, A.P.: Robust object recognition in an unstructured environment. In: Intelligent Autonomous Systems 12. Advances in Intelligent and Soft Computing Series, Springer (2012)

26. Martinez-Martin, E., del Pobil, A.P.: Robust Motion Detection in Real-Life Scenarios. Springerbr edn. SpringerBriefs in Computer Science. Springer London, London (2012)

27. Bay, H., Ess, A., Tuytelaars, T., Van Gool, L.: Speeded-up robust features (surf). CVIU 110(3) (2008) 346-359 